\title{
Exploring control parameters of two photon processes in solutions ${ }^{\#}$
}

\author{
DEBABRATA GOSWAMI ${ }^{\mathrm{a}, *}$ and AMIT NAG ${ }^{\mathrm{a}, \mathrm{b}}$ \\ ${ }^{a}$ Department of Chemistry, Indian Institute of Technology Kanpur, Kanpur 208 016, India \\ ${ }^{b}$ Present address: Department Chemie und CeNS Ludwig-Maximilians-University, Munich, \\ Butenandtstr.11, HausE, 81377 Munich, Germany \\ e-mail: dgoswami@iitk.ac.in
}

\begin{abstract}
Two-photon microscopy depends extensively on the two-photon absorption cross-sections of biologically relevant chromophores. High repetition rate (HRR) lasers are essential in multiphoton microscopy for generating satisfactory signal to noise at low average powers. However, HRR lasers generate thermal distortions in samples even with the slightest single photon absorption. We use an optical chopper with HRR lasers to intermittently 'blank' irradiation and effectively minimize thermal effects to result in a femtosecond z-scan setup that precisely measures the two-photon absorption (TPA) cross-sections of chromophores. Though several experimental factors impact such TPA measurements, a systematic effort to modulate and influence TPA characteristics is yet to evolve. Here, we present the effect of several control parameters on the TPA process that are independent of chromophore characteristics for femtosecond laser pulse based measurements; and demonstrate how the femtosecond laser pulse repetition rate, chromophore environment and incident laser polarization can become effective control parameters for such nonlinear optical properties.
\end{abstract}

Keywords. Nonlinear optical control; two photon absorption; two photon absorption cross-section; polarization; pulse repetition rate; nonlinear index of refraction.

\section{Introduction}

Two-photon absorption (TPA) and in general multiphoton absorption is a quantum process, where an atom or a molecule absorbs two or more photons simultaneously. ${ }^{1}$ Typically, TPA processes require materials with large TPA cross-sections $\left(\sigma_{2}\right)$, which are directly related to the imaginary part of the second order hyperpolarizability $[\operatorname{Im} \gamma(-\omega, \omega, \omega,-\omega)]$.

A measure of the two-photon cross-sections (TPCS), thus characterizes TPA. Study of TPCA of molecules have pioneered technologies, such as, two photon imaging microscopy, ${ }^{2}$ photodynamic therapy, ${ }^{3}$ optical limiting, ${ }^{4}$ all-optical switching and signal processing, ${ }^{5}$ etc. For most of these applications, design and synthesis of molecules with large TPCS measured through absorption or fluorescence has received a lot of attention. ${ }^{6-8}$ Modulation in TPCS is achieved with increased electron delocalization in molecules by synthetic design strategies. ${ }^{9-13}$ However, in addition to the challenges in synthetic skill, research in the design and synthesis of molecules with high TPCS faces the bottleneck of laser interaction characteristics. Wavelength tunable TPCS of molecules is intricate and further laser control parameters are certainly desirable, which essentially amounts

\footnotetext{
\#Dedicated to Prof. N Sathyamurthy on his 60th birthday

*For correspondence
}

to control of TPA and related processes. Typical control parameters or 'control knobs' of the interacting laser pulse, such as, the temporal shape, frequency content, intensity, polarization, etc. are of great interest due to their generic applicability irrespective of the system concerned. However, two-photon processes are difficult to control-more so in the condensed phase and most model calculations have failed to come up with simple control parameters that would have predictable results.

Use of femtosecond lasers have provided a large impetus in this overall quest as femtosecond pulses offer the advantage of high peak intensity at fairly low average powers to induce nonlinear effects without any sample damage. Previous efforts on the effect of pulsewidth on nonlinear processes have shown that, femtosecond pulses ensure that none of the system dependent relaxation processes can interfere with the nonlinear optical (NLO) process being measured. The same system relaxation argument also implies that these ultrashort pulses should be well-separated in time to ensure that the effect of each pulse can be treated independently. Nevertheless, in biological research, particularly in the field of two-photon and multiphoton microscopy and imaging, high repetition rate (HRR) lasers are essential for having satisfactory signal to noise at low average powers. The HRR lasers can generate thermal distortions in samples even with the slightest single photon absorption. We have developed a 
femtosecond open aperture z-scan (OAZS) setup using an optical chopper with HRR lasers ('blanking') that effectively minimizes thermal as well as small linear absorption effects and precisely measures $\sigma_{2}$ of chromophores. Using femtosecond HRR lasers, therefore, we accurately measure $\sigma_{2}$ values with high sensitivity for various chromophores. ${ }^{9-13}$ This also establishes the fact that pulse-to-pulse repetition rate plays a significant role in TPA and in this paper we present, effect of pulse repetition rate as an important control parameter for such NLO processes. By keeping all the other experimental parameters the same as above, experiments with closed aperture z-scan (CAZS) by $20 \%$ opening of the aperture in the far field enabled us to calculate $\mathrm{n}_{2}$.

Two-photon processes are affected by several external factors. The first and foremost one is the effect of the surrounding solvent medium in TPA processes. There have been several indications in the literature, ${ }^{14-20}$ that effect of solvent polarity can play an important role in the modulation of TPCS of the chromophores. Thus, though molecular design has been the most dominant scheme for achieving large $\sigma_{2}$ values, solvent properties also change TPA properties. We also discuss some of our efforts towards a systematic study of solvent effects, which was aimed towards using controlling external environmental effects on TPA, and last but not the least, we present the effect of incident laser polarization on TPA. Typically, all the TPA measurements are performed with the linearly polarized light. However, our polarization studies on TPA indicate that there is a preference towards the linear component of the incident laser which is opposite to that of the single photon absorption studies.

Thus, in this paper we show the effect of several control parameters on the TPA process that are independent of chromophore characteristics for femtosecond laser pulse based measurements, and demonstrate how the femtosecond laser pulse repetition rate, chromophore environment and incident laser polarization can become effective control knobs for such an NLO process.

\section{Experimental details}

We have chosen a range of Rhodamine (Rh) dyes for our experiments (figure 1). We also use Fluorescein and Coumarin-30 as two additional two-photon dyes for near-IR wavelengths. Rhodamines are well known xanthenes dyes, which have been extensively used for many widespread applications in single-molecule detection, ${ }^{21}$ DNA-sequence determination, ${ }^{22}$ fluorescence labelling, ${ }^{23}$ etc. due to their strong fluorescence over the visible spectral region. Molecular geometries of $\mathrm{Rh}$ dyes are well-known ${ }^{24,25}$ and indicate that all the structures are noncentrosymmetric. Fluorescein has been used as a standard dye for calibration in the TPF techniques. ${ }^{26}$ Coumarin-30 is also recently used in an optimal feedback control study of two-photon fluorescence. ${ }^{27}$ Single photon fluorescence (SPF) experiments are done on the popular single photon near-IR dyes, IR125 and IR144. ${ }^{28,29}$ All these dyes are very relevant for microscopy and our efforts are to demonstrate laser parameter induced control on the two-photon processes as compared to their corresponding single-photon case as the typical selection rules and pathways of molecular transitions for two-photon excitation may be different from that of the single-photon excitation. Such a systematic control approach should work irrespective of the chromophore characteristics.

Spectroscopy grade Rh6G (dye content: 99\%), RhB (dye content: 99\%), and Rh101 (dye content: 99\%), Flourescein, Coumarin-30 IR125 and IR144 dyes were purchased from Sigma-Aldrich and used without further purification. All the organic solvents: chloroform $\left(\mathrm{CHCl}_{3}\right)$, dichloromethane (DCM), dimethylformamide (DMF) and methanol $(\mathrm{MeOH})$ used for the experiments are of HPLC-grade quality and their UV-Vis spectra were obtained to ensure purity. For pure water, we used the double-distilled $18 \mathrm{M} \Omega$ Millipore quality.

Linear absorption spectra for all the dyes in various solvents at $10^{-5} \mathrm{M}$ concentration are measured in $1 \mathrm{~cm}$ long quartz cuvette with a diode-array spectrophotometer (Agilent-8453) after subtracting the cuvette and the respective solvent contribution. However, for all the nonlinear measurements, a concentration of $0.005 \mathrm{M}$ is used. Purity of the dyes is a very important factor, as impurities can severely affect their photo-physics through competitive processes. However, given our dye purity, such effects can only add an error of at most $1 \%$ to our experimental data. IR125 and IR144 dyes were prepared at the concentration of $10^{-5} \mathrm{M}$ for polarization dependent SPF measurements. All our measurements of NLO and the $\sigma_{2}$ values were performed by using the standard open-aperture z-scan (OAZS) technique. ${ }^{30-34}$ We have taken the known $\sigma_{2}$ value of $1 \mathrm{mM}$ Rhodamine-6G in methanol as a reference ${ }^{35,36}$ for calibrating our measurements.

The NLO measurements were performed at nonresonant laser wavelengths. We used two different laser systems for the experiments (figure 2). In the first case, a mode-locked Coherent MIRA Ti:Sapphire laser (Model 900) pumped by the Coherent Verdi frequency-doubled Nd:Vanadate laser was used to provide high repetition rate (HRR) femtosecond laser pulses with low average power. MIRA-900 operates at $76 \mathrm{MHz}$ repetition rate and is wavelength tunable 

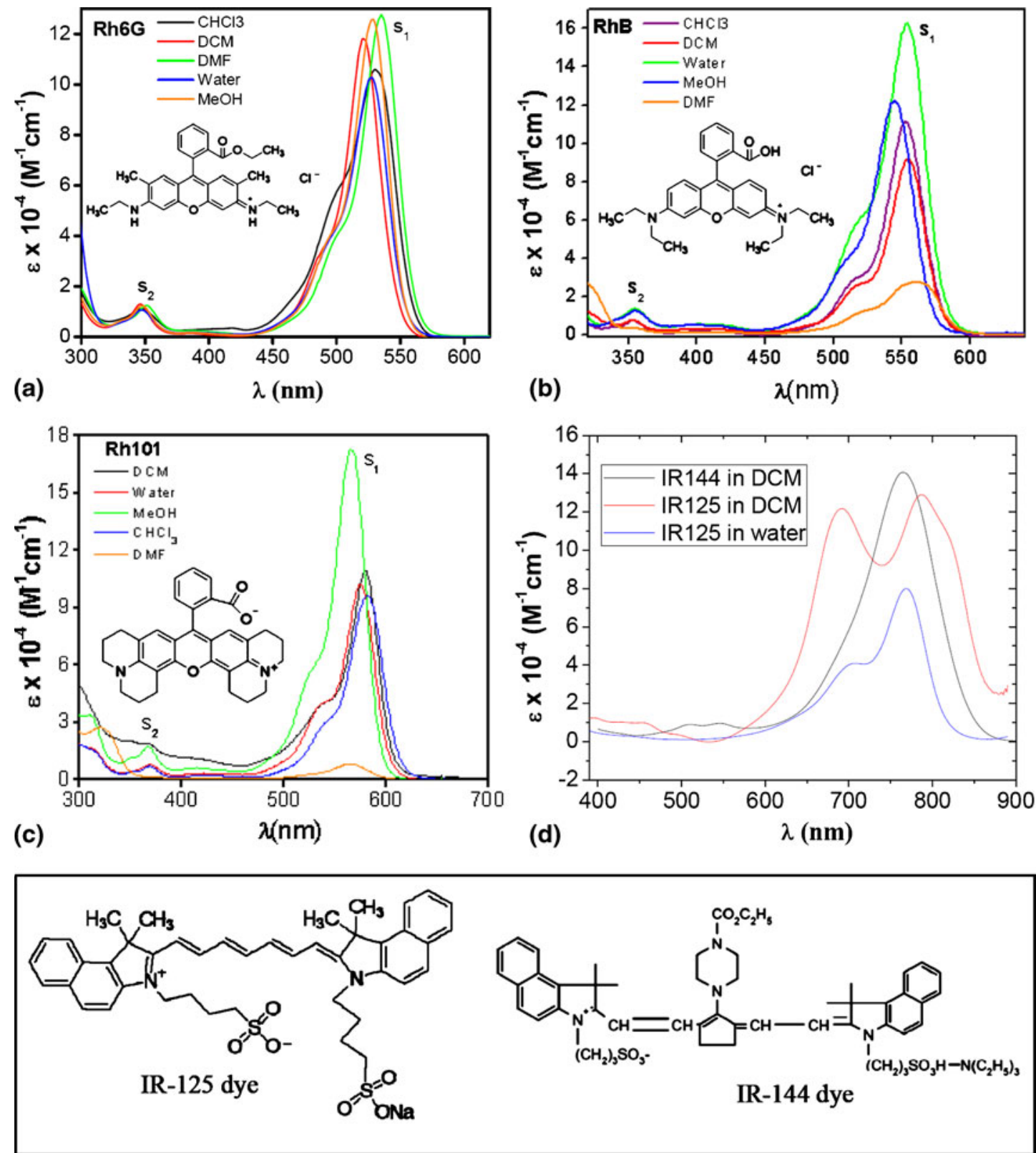

(e)

Figure 1. Linear absorption spectra of the Rhodamine dyes in different solvent medium: (a) Rh6G, (b) $\mathrm{RhB}$ and (c) Rh101 indicating the $\mathrm{S} 1$ and $\mathrm{S} 2$ transition peaks. A concentration of $10^{-5} \mathrm{M}$ is used for measuring the spectra. Corresponding dye structures are also given in the inset. (d) Linear absorption spectra of IR125 and IR144 in DCM. Molecular structures of IR125 and IR144 are shown in (e).

in $730 \mathrm{~nm}$ to $900 \mathrm{~nm}$ with an average pulsewidth of 120 fs. TPA studies with z-scan method with HRR lasers can be corrupted by the thermal effects arising from coupled nonlinear refraction even with femtosecond pulses in completely transparent two-photon samples. So, our nonlinear transmission measurements with the HRR laser employed a lock-to-clock mechanical chopper MC1000A (Thorlabs Inc.) with variable frequency at $50 \%$ duty cycle for blanking. The optimized frequency of this chopper is attributed to the minimized cumulative thermal effect of the sample by HRR pulses at excitation wavelength. TPA measurements under such chopped condition coincide with the experimental results from our second experimental set-up, which instead of using a chopper uses a low repetition rate (LRR) commercial multipass amplified Ti:sapphire laser (ODIN, Quantronix Inc.), pumped by the Coherent $120 \mathrm{~ns}$ Nd-YAG Corona laser to routinely 


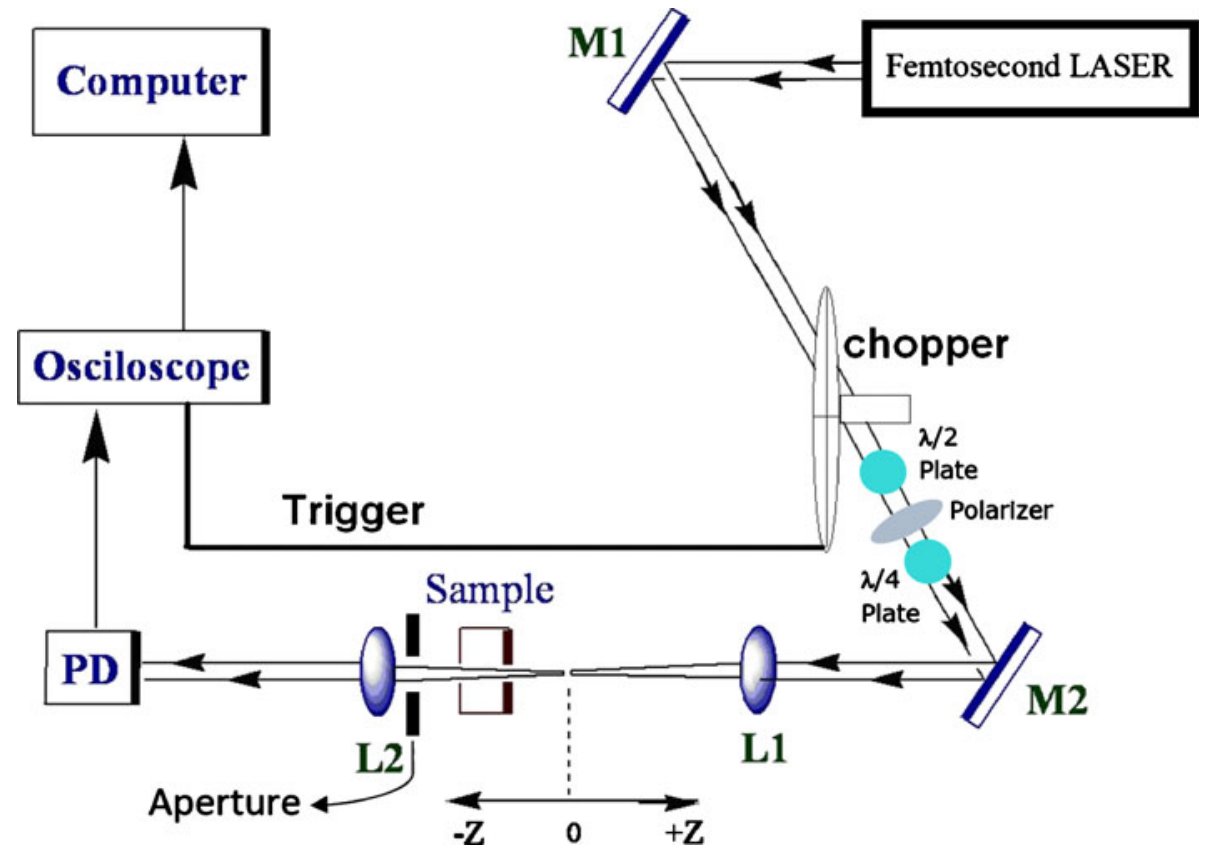

Figure 2. Experimental set-up for the measurement of two-photon absorption crosssection: The aperture after the sample determines whether it is an open-aperture or close-aperture experimental condition. The half-wave plate polarizer combination is for intensity modulation and the quarter-wave plate is for the polarization dependent studies. Use of chopper before the sample for the HRR laser distinguishes it from a conventional z-scan open-aperture set-up (see text for more details).

generate $50 \mathrm{fs}$ pulses at $806 \mathrm{~nm}$ with $1 \mathrm{kHz}$ repetition rate. $^{37}$

The shortest possible MIRA pulses are $118 \mathrm{fs}$ while that of ODIN are $34 \mathrm{fs}$. For OAZS experiments, the pulses in both the cases are focused into the sample cell using a $20 \mathrm{~cm}$ focal length lens, which can give peak powers to $\geq 1 \mathrm{GW} / \mathrm{cm}^{2}$ at focus. After appropriate attenuation, the laser intensity at the focus in both the cases is $\sim 10 \mathrm{MW} / \mathrm{cm}^{2}$. Gaussian beams were used for all the experiments without any spatial filtering. The $1 \mathrm{~mm}$ long sample cell (quartz cuvette) satisfies the condition that the cell-length is less than the Rayleigh range of the focusing lens as the Rayleigh range in our set-up is $>1 \mathrm{~mm}$ in entire wavelength tuning range of $730 \mathrm{~nm}$ to $900 \mathrm{~nm}$ (e.g., Rayleigh range at $770 \mathrm{~nm}$ is $1.2 \mathrm{~mm}$ as the beam waist at focus is $17 \mu \mathrm{m}$ ). We scanned the sample through the focal point of the lens using a motorized translation stage (Newport Inc. model ESP-300), which has a minimum step-size of $0.1 \mu \mathrm{m}$. This allows for a smooth intensity scan of the sample. The transmitted beam through the sample is focused with a $7.5 \mathrm{~cm}$ focal length lens into a UV-enhanced amplified silicon photo detector (Thorlabs DET210). The peakto-peak value from the photodiode is measured with an oscilloscope (LeCroy WaveRunner Model LR6100R), which is triggered by the chopper frequency. The delay stage and the oscilloscope are interfaced with the computer using a GPIB card (National Instruments Inc.) and the data is acquired using LabVIEW programming. Closed aperture z-scan (CAZS) experiments are also performed to calculate $n_{2}$ by $20 \%$ opening of the aperture in the far field, by keeping all the other experimental parameters intact.

\section{Results and discussion}

For TPA processes, nonlinear absorption coefficient values $(\beta)$, can be obtained by curve fitting to the observed open-aperture transmitted intensity, $T(z)$, for a sample of length $L$, measured as a function of the sample position with respect to the distance $(z)$ from the focal point $\left(z_{0}\right)$ as: ${ }^{37} T(z)=1-\beta I_{0} L / 2^{\frac{3}{2}}\left(1+\frac{z^{2}}{z_{0}^{2}}\right)$. Thus, $\sigma_{2}$ can be determined by using the relationship:

$$
\sigma_{2}=\beta \hbar v \cdot 10^{3} / N_{A} d,
$$

$\sigma_{2}$ can also be calculated by the alternative slope method, ${ }^{35}$ which involves plotting the inverse of transmitted photon signal in the z-scan experiment with 

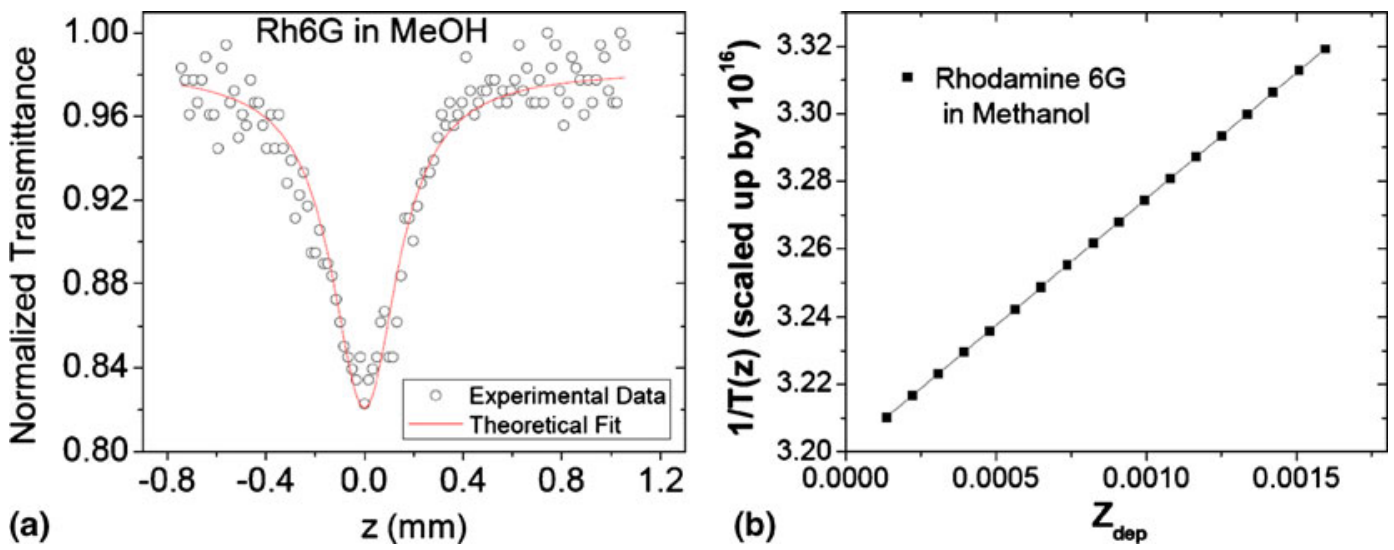

Figure 3. (a) Open aperture Z-scan of Rhodamine-6G fitted to the theoretical model given by equation (1) (solid line). (b) Plot of the inverse of the raw Z-scan scan data with the modified z-dimension, $Z_{\text {dep }}$ as derived in equation (2) for the slope method which result in the same TPA $\sigma_{2}$ values as from $(\mathbf{a})$.

respect to the modified $\mathrm{z}$-scale dimension that can be derived to be: $1 / T(z)=0.66 d \sigma_{2} \mu N_{A} Z_{d e p}(z) / \lambda \tau+$ $1 / I_{0}$, where, modified z-scale dimension is, $Z_{\text {dep }}(z)=$ $\left[\tan ^{-1}\left((z+L / 2) / z_{0}\right)-\tan ^{-1}\left((z+L / 2-L / \mu) / z_{0}\right)\right]$, with $\mu$ as the refractive index of the sample and $\tau$ as the full width half maximum pulsewidth of the laser pulse at centre wavelength $\lambda$. If the measured slope for the plot is $M$, the $\sigma_{2}$ (in units of GM) can be represented as:

$$
\sigma_{2}=M \lambda \tau \cdot 10^{50} / 0.66 d \mu N_{A} .
$$

Equations (1) and (2) are the two approaches that we use for getting the TPA $\sigma_{2}$ values for all OAZS measurements. Figure 3 shows correspondence of the two approaches for OAZS data collected with our $1 \mathrm{kHz}$ ODIN laser set-up to result in the calibrated $\sigma_{2}$ value of $16.1 \mathrm{GM}$ at $806 \mathrm{~nm} .{ }^{36}$

The importance of the femtosecond pulses to induce the TPA is shown in figure 4a. However, HRR pulses from the Mira-900 setup as explained in the earlier section does start showing up as distortion to the fit which gives rise to the best match to the calibrating case of $\mathrm{Rh} 6 \mathrm{G}$ in $\mathrm{MeOH}$ as was seen in the case of the $\mathrm{kHz}$ system though use of a chopper can recover the situation (figure $4 b$ ).

The successful blanking of HRR laser to minimize thermal effects as presented in figure $4 \mathrm{~b}$ by using a $50 \%$ duty-cycle chopper with $1 \mathrm{~ms}$ on and $1 \mathrm{~ms}$ off can be
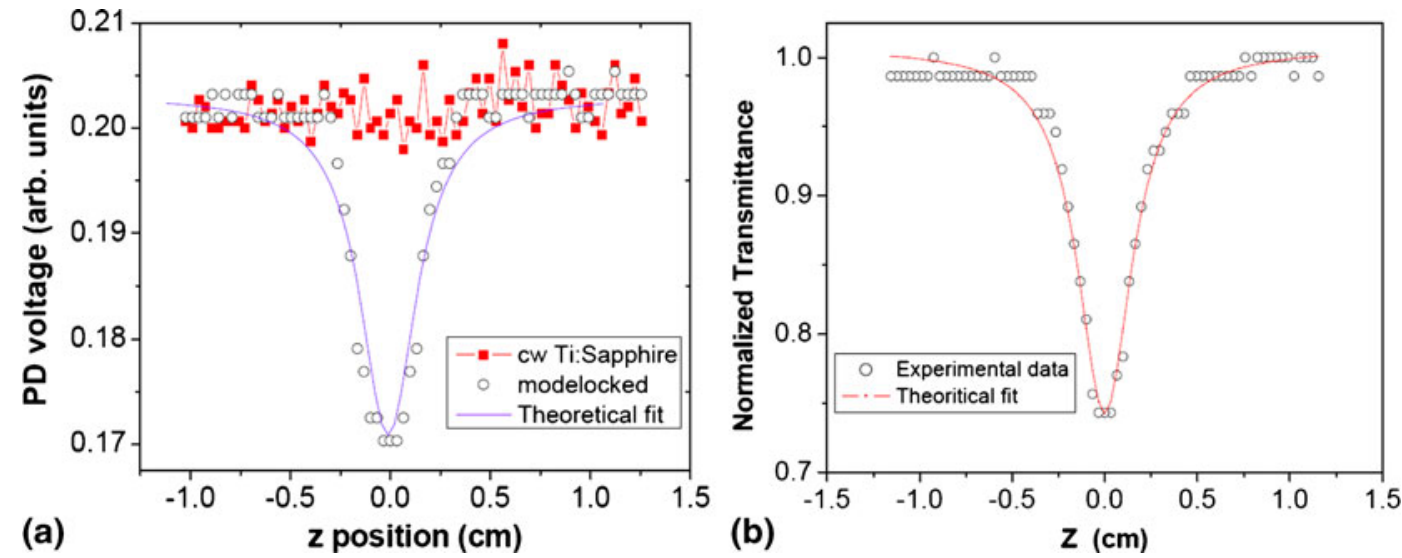

Figure 4. (a) Comparison of HRR femtosecond laser versus $\mathrm{cw}$ laser induced $\mathrm{z}$-scan data at identical sample concentration and laser average powers without the use of chopper at $780 \mathrm{~nm}$. Femtosecond pulses are critical for TPA. Deviation from theoretical fit for even such non-absorbing sample for HRR laser at $780 \mathrm{~nm}$ is also visible. (b) Open aperture z-scan trace of Rh6G in MeOH with HRR laser set-up with the use of chopper to minimize thermal effects at $780 \mathrm{~nm}$. Theoretical fitting works perfectly within the experimental error-bar under this chopped condition. 

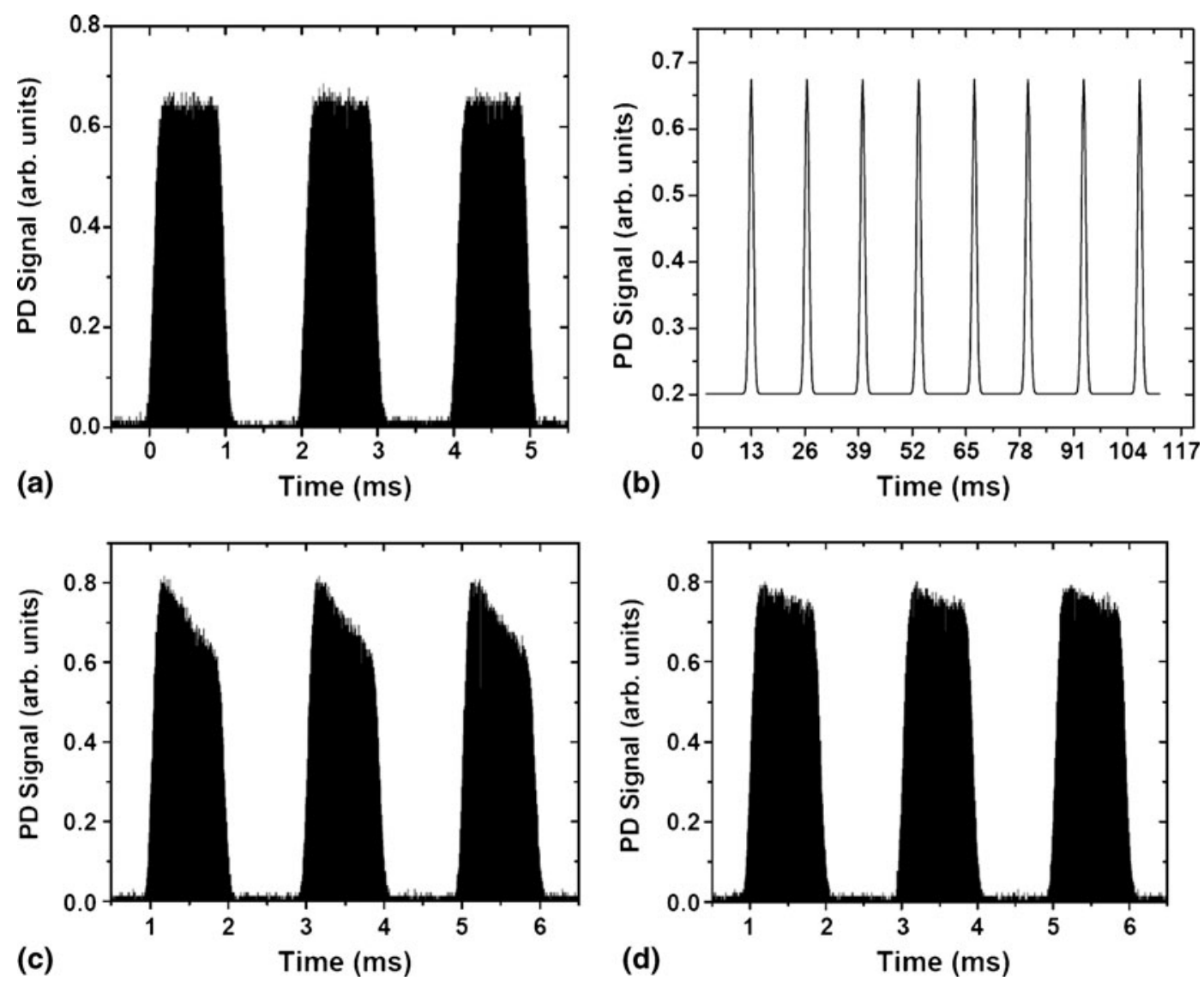

Figure 5. (a) Chopper 'blanking' at $500 \mathrm{~Hz}$ wherein the mode-locked pulses are present for $1 \mathrm{~ms}$ and absent for $1 \mathrm{~ms}$ with an exact 50\% duty cycle. (b) Mode-locked train of pulses that are $13.6 \mathrm{~ns}$ apart is unaffected within the chopped area. (c) Effect of linear absorption on chopped signal as the focal point $(\mathrm{z}=0)$ approaches for a $1 \mathrm{~cm}$ sample cell. (d) The same case as in (c) except that the sample cell is $1 \mathrm{~mm}$ long. Thermal effects are minimized as compared to (c). Since we measure peak to peak values on the oscilloscope, our data distortion is further minimized.

further understood from figure 5, where the peak-topeak data-acquisition principle for TPA experiments are justified for minimizing thermal effect.

In addition to such experimental details on pulse repetition affecting the TPA measurements, the peak power of the pulses can also have other important NLO consequences. Thus, for instance, using CAZS experiments with HRR femtosecond laser (figure 6a), both Rh6G and RhB shows negative $n_{2}$ in all the solvents with a characteristic pre-focal transmittance maximum (peak) followed by a post focal transmittance minimum (valley). The highest value of $n_{2}$ is obtained from $R h B$ in $\mathrm{CHCl}_{3}$ as $-3.5 \times 10^{-13} \mathrm{~cm}^{2} /$ Watt. We even measured the nonlinearity of the neat solvents used in these experiments with the CAZS and found that except for $\mathrm{MeOH}$, all the other solvents have no measurable nonlinearity at identical laser powers and same experimental conditions. $\mathrm{MeOH}$ has small negative $\mathrm{n}_{2}$ values ${ }^{38}$ when measured with HRR lasers. Next, we measured these $n_{2}$ values with our $1 \mathrm{kHz}$ amplified laser (ODIN) also. In fact, even very low average laser power from the amplified laser also generated positive $\mathrm{n}_{2}$ (figure $6 \mathrm{~b}$ ). Experiments performed by changing the laser repetition rate using $120 \mathrm{~ns}$ Corona pulses at $532 \mathrm{~nm}$, whose repetition rate was changed from 1 to $25 \mathrm{kHz}$, however, show negative $n_{2}$ values just like the measurements from the HRR lasers. The important point to note here is that the off-time of $\sim 1 \mathrm{~ms}$ available for the $\sim 50 \mathrm{fs}$ ODIN laser at $1 \mathrm{kHz}$ helps to best minimize the thermal effects as compared to the $120 \mathrm{~ns}$ pulses at 1 to $25 \mathrm{kHz}$ or the HRR laser. Thus, we conjecture that the sign of $\mathrm{n}_{2}$ is highly influenced by thermal effects.

So far we have explored the effect of many possible pulse variables associated with femtosecond laser based NLO studies to show their intricate connection to such measurements. The most important of the ones discussed here are the intensity, pulsewidth and repetition rate, under the implicit assumption that we are always trying to operate at wavelengths that have minimum linear effects. The wavelength parameter of the 

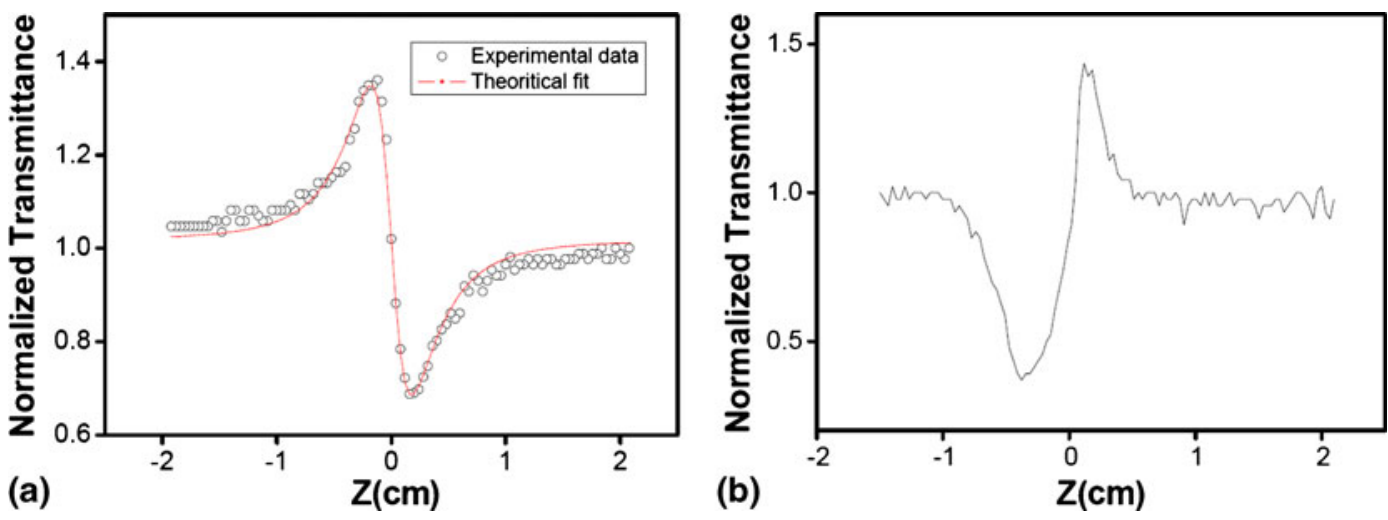

Figure 6. CAZS plot of Rh6G in $\mathrm{MeOH}$ at $806 \mathrm{~nm}$ by (a) $76 \mathrm{MHz}$ oscillator showing negative $\mathrm{n}_{2}$, and (b) $1 \mathrm{kHz}$ laser Odin showing positive $\mathrm{n}_{2}$.

laser is also a nontrivial parameter as in general, for centrosymmetric molecules, TPA is forbidden when tuned to the transitions at one-half of the excita- tion frequencies. However, for non-centrosymmetric molecules due to symmetry relaxations, the single photon absorption (SPA) peaks and energy corresponding
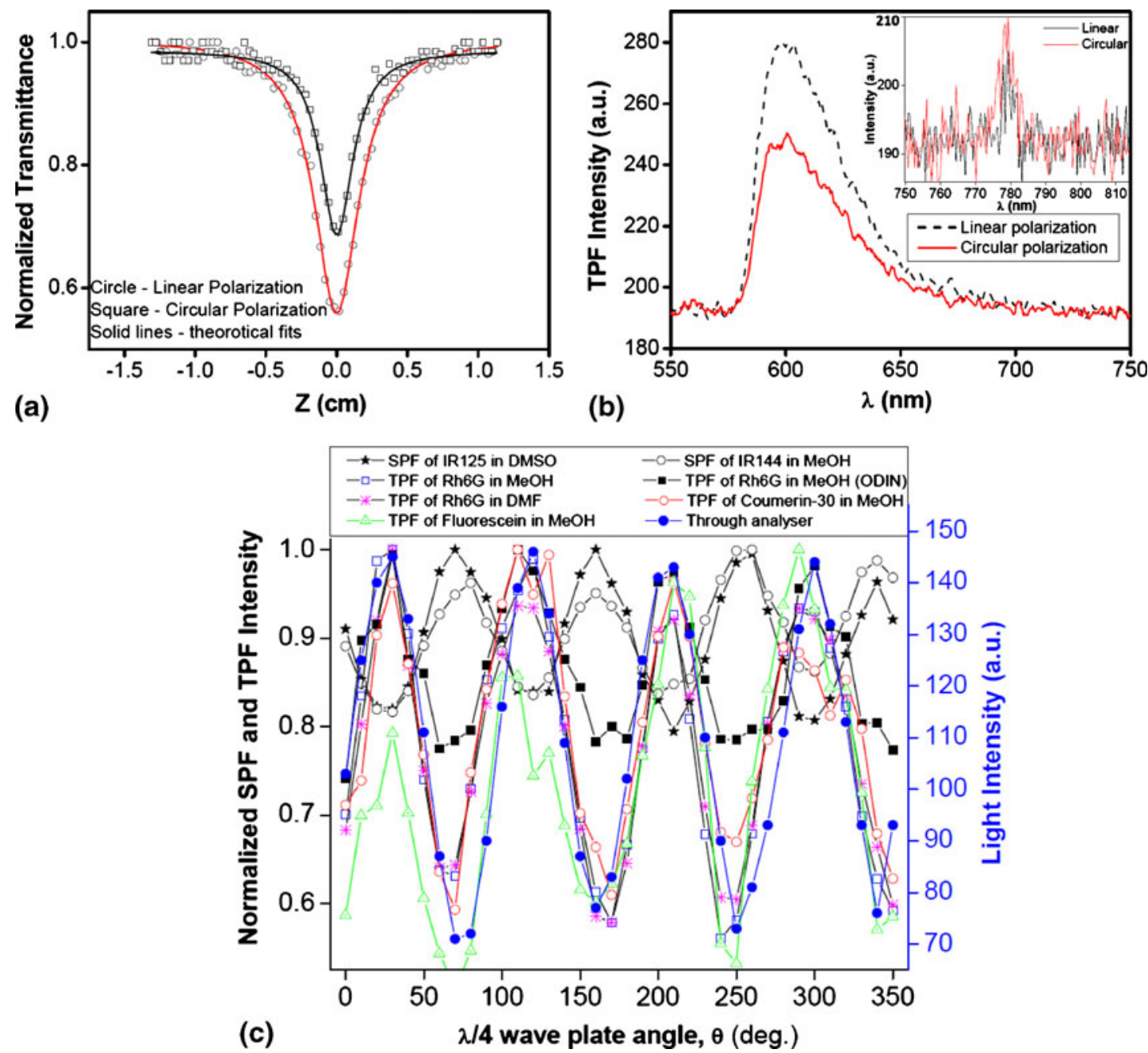

Figure 7. (a) Open aperture $\mathrm{z}$-scan plots of $\mathrm{Rh} 6 \mathrm{G}$ in $\mathrm{MeOH}$ at $810 \mathrm{~nm}$ for linear and circular polarization of the amplified $\mathrm{kHz}$ laser Odin. (b) TPF spectra of Rh6G dye for linear and circular polarization, when excited at $780 \mathrm{~nm}$ from high repetition rate Mira laser. Inset in the graph shows the 780 beam spectra at two polarizations. (c) Variation of TPF intensity of different fluorescent dyes with quarter wave plate rotations. The modulation exactly follows the linear component of the beam. 
to TPCS may coincide. Nevertheless, though intensity, wavelength and pulsewidth are connected parameters in terms of the transition energy of the concerned TPA molecules, the repetition rate is an independent parameter. Next, let us consider another independent laser parameter: laser polarization. Figure 7 shows that the TPA and the corresponding two-photon fluorescence (TPF) of Rh6G, Fluorescein and Coumarin-30 are highly modulated by the incident laser light polarization under different conditions. These results show that such laser polarization effects manifest irrespective of the exact nature of the sample, its surroundings and/or pulse repetition rate or pulse-width effects. We find that the polarization modulations also exist in SPA and the corresponding SPF and a comparison to the polarization dependent SPF study was made possible with the help of linear absorption studies with IR125 and IR144 dyes. The incident laser polarization was varied by a quarter-wave plate ( $\lambda / 4$ plate) from linear through elliptical to circular and vice-versa; while a half-wave plate ( $\lambda / 2$ plate) was used to change the laser polarization from horizontal to vertical and vice-versa. Such results were compared with the changes in the component of light for both $\lambda / 4$ and $\lambda / 2$ plates by using a Glan-Laser polarizer without any sample. Interestingly, the TPA or TPF modulation with laser polarization shows suppression with circularly polarized light and this behaviour maps exactly the linear component of the laser beam through an analyser. However, this two-photon behaviour is completely out of phase with that of the SPF modulation with polarization (figure 7). This change in TPA intensity with laser polarization resulting in circular-linear dichroism (CLD) can be potentially useful in structure determination of aligned biomolecules. ${ }^{39}$

As expected, there is no difference in the TPA intensity between right and left circularly polarized light for such non-chiral isotropic samples, though these results definitively show an overall effect of polarization on
$\mathrm{NLO}^{30}$ and further work on this is in progress in our laboratory.

Finally, we discuss the effect of solvent on the chromophore TPA activity. Solvent dependent TPA spectra of the dyes were measured with OAZS method and compared to their respective single-photon spectra at equivalent energies. A comparison between figures 1 and 8 show that local environment effect is more drastic in case of TPA as compared to the single photon case. In the TPA spectra, relative peak intensities and positions are highly solvent dependent, which could be a result of vibronic couplings that depend on solvent environment.

Our results (figure 8) indicate that the relative intensity of the TPA peaks in non-polar solvents $\mathrm{CHCl}_{3}$ and DCM are very different while that of $\mathrm{MeOH}$ and DMF are similar. In fact, in polar solvents, the relative intensities of the TPA peaks follow same trend as the SPA spectra. Though it can be conjectured that the solvents change the TPA properties of the chromophores through a change in the dielectric constant of the medium, which in turn can change the chromophore electronic structure by inducing geometrical distortions like a change in the torsional angle between the aromatic rings of the chromophores, it is not possible to specifically identify how the solvent is affecting the vibronic coupling mediated TPA process here. In general, we have shown that the solvent polarity does affect the linear and nonlinear properties of the chromophores. However, other factors, such as, hydrogen bonding, viscosity, solutesolvent interactions, and solubility, should also be considered for solving this complex problem.

\section{Conclusions}

We have shown the effect of several control parameters on the TPA processes. While the laser peak power is critical and often the threshold condition in the generation of the NLO process in question, several other
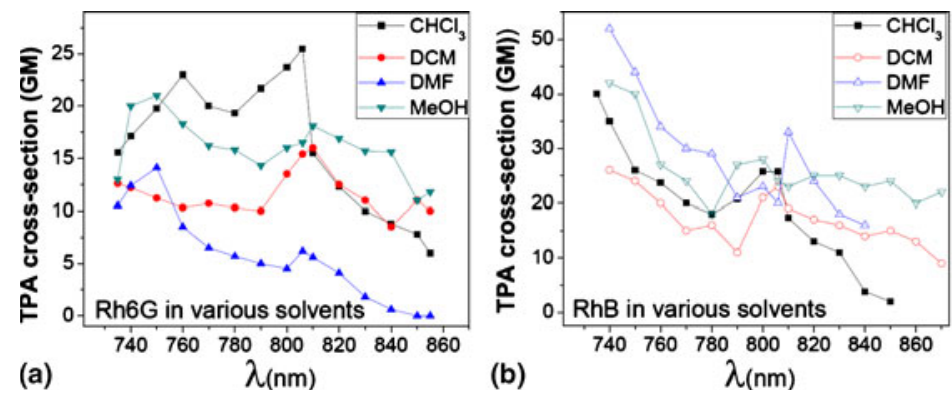

Figure 8. Solvent effect study of $\mathrm{CHCl3}$, DCM, DMF and $\mathrm{MeOH}$ on the TPCS of various Rhodamine dyes: (a) Rh6G, (b) RhB and (c) Rh101 through OAZS technique at various wavelengths using chopped tunable MIRA-900. 
laser characteristics and the chromophore environment could be important enough to be used as NLO control parameters. We have used OAZS and CAZS experiments that are independent of chromophore characteristics for femtosecond laser pulse-based measurements, and demonstrated how the femtosecond laser pulse repetition rate, chromophore environment and incident laser polarization can become effective control knobs.

\section{Acknowledgements}

DG thanks Ministry of Communication and Information Technology (MCIT), Swarnajayanti Fellowship of the Department of Science and Technology (DST) (India) and International SRF Program of Wellcome Trust (UK) for financial grants.

\section{References}

1. Goppert-Mayer M 1931 Ann. Phys. 9273

2. Denk W, Strickler J H and Webb W W 1990 Science 248 73

3. Arnbjerg J, Jimenez-Banzo A, Paterson M J, Nonell S, Borrell J I, Christiansen O and Ogilby P R 2007 J. Am. Chem. Soc. 1295188

4. Lin T C, He G S, Zheng Q and Prasad P N 2006 J. Mat. Chem. 162490

5. Stegeman G I 2001 Handbook of optics IV: Fiber optics \& nonlinear optics $2^{\text {nd }}$ ed.; M Bass, J M Enoch, E W V Stryland, and W L Wolfe, (eds.), New York: McGrawHill, Chapter 21

6. Albota M, Beljonne D, Brédas J L, Ehrlich J E, Fu J-Y, Heikal A A, Hess S E, Kogej T, Levin M D, Marder S R, McCord-Maughon D, Perry J W, Röckel H, Rumi M, Subramaniam G, Webb W W, Wu X-L and Xu C 1998 Science 2811653

7. Kogej T, Beljonne D, Meyers F, Perry J W, Marder S R and Bredas J L 1998 Chem. Phys. Lett. 2981

8. Cho B R, Son K H, Lee S H, Song Y-S, Lee Y-K, Jeon S-J, Choi J H, Lee H and Cho M 2001 J. Am. Chem. Soc. 12310039

9. Rath H, Sankar J, PrabhuRaja V, Chandrashekar T K, Nag A and Goswami D 2005 J. Am. Chem. Soc. 127 11608

10. Das S, Nag A, Goswami D and Bharadwaj P K 2006 J. Am. Chem. Soc. 128402

11. Misra R, Kumar R, Chandrashekar T K, Suresh C H, Nag A and Goswami D 2006 J. Am. Chem. Soc. 128 16083

12. Misra R, Kumar R, Chandrashekar T K, Nag A and Goswami D 2006 Org. Lett. (Letter) 8629

13. Rath H, PrabhuRaja V, Chandrashekar T K, Nag A, Goswami D and Joshi B S 2006 Org. Lett. (Letter) 8 2325
14. Luo Y, Norman P, Macak P and Ågren H 2000 J. Phys. Chem. A 1044718

15. Wang C K, Zhao K, Su Y, Yan R, Zhao X and Luo Y 2003 J. Chem. Phys. 1191208

16. Frediani L, Rinkevicius Z and Ågren H 2005 J. Chem. Phys. 122 244104-1

17. Zhao K, Ferrighi L, Frediani L, Wang C K and Luo Y 2007 J. Chem. Phys. 126 204509-1

18. Woo H Y, Liu B, Kohler B, Korystov D, Mikhailovsky A and Bazan G C 2005 J. Am. Chem. Soc. 12714721

19. Fitilis I, Fakis M, Polyzos I, Giannetas V, Persephonis P, Vellis P and Mikroyannidis J 2007 Chem. Phys. Lett. 447300

20. Yan Y, Li B, Liu K, Dong Z, Wang X and Qian S 2007 J. Phys Chem. A 1114188

21. Keller R A, Ambrose W P, Goodwin P M, Jett J H, Martin J C and Wu M 1996 Appl. Spectrosc. 50 $12 \mathrm{~A}$

22. Bergot B J, Chakerian V C, Connell R J, Eadie S S, Fung N H, Davi L, Lee G, Mechen S M and Woo S L 1996 Biotechnol. Adv. 1485

23. Fontaine M, Elmquist W F and Miller D W 1996 Life Sci. 591521

24. Watanabe H, Hayazawa N, Inouye Y and Kawata S 2005 J. Phys Chem. B 1095012

25. Sarkar J, Chowdhury J, Pal P and Talapatra G B 2006 Vib. Spectrosc. 4190

26. Albota M, Xu C and Webb W W 1998 Appl. Opt. 37 7352

27. Zhang S, Sun Z, Zhang X, Xu Y, Wang Z, Xu Z and Li R 2005 Chem. Phys. Lett. 415346

28. Takechi K, Sudeep P K and Kamat P V 2006 J. Phys Chem. B 11016169

29. Yu A, Tolbert C A, Farrow D A and Jonas D M 2002 J. Phys Chem. B 1069407

30. Nag A and Goswami D 2010 J. Chem. Phys. 132 0154508

31. Nag A and Goswami D 2009 J. Photochem. Photobiol. A: Chemistry 206188

32. Sheik-Bahae M, Said A A, Wei T, Hagan D J and Van Stryland E W 1990 IEEE J. Quantum Electron. 26760

33. Van Stryland E W and Sheik-Bahae M 1998 Characterization techniques and tabulations for organic nonlinear materials, (eds.): M G Kuzyk, C W Dirk, Marcel Dekker, Inc. pp. 655

34. Goswami D 2006 Opt. Commun. 261158

35. Sengupta P, Balaji J, Banerjee S, Philip R, Kumar G R and Maiti S 2000 J. Chem. Phys. (Communication) 112 9201

36. Tian P and Warren W S 2002 Optics Lett. 271634

37. Nag A, De A K and Goswami D 2009 J. Phys. B: At. Mol. Optical Phys. 42065103

38. Nag A, Singh S and Goswami D 2006 Chem. Phys. Lett. 430420

39. Wanapun D, Wampler R D, Begue N J and Simpson G J 2008 Chem. Phys. Lett. 4556 